Gut and Liver, Vol. 14, No. 3, May 2020, pp. 387-394

\title{
Factors of Endoscopic Ultrasound-Guided Tissue Acquisition for Successful Next-Generation Sequencing in Pancreatic Ductal Adenocarcinoma
}

\author{
Jae Keun Park ${ }^{1}$, Ji Hyeon Lee ${ }^{2}$, Dong Hyo Noh², Joo Kyung Park², Kyu Taek Lee ${ }^{2}$, Jong Kyun Lee ${ }^{2}$, Kwang Hyuck Lee ${ }^{2,3}$, \\ Kee-Taek Jang ${ }^{4}$, and Juhee Cho ${ }^{3,5,6}$ \\ ${ }^{\prime}$ Digestive Disease Center and Research Institute, Department of Internal Medicine, Soonchunhyang University School of Medicine, Bucheon, \\ ${ }^{2}$ Department of Medicine, Samsung Medical Center, Sungkyunkwan University School of Medicine, ${ }^{3}$ Department of Clinical Research Design \\ and Evaluation, Samsung Advanced Institute for Health Sciences \& Technology (SAIHST), Sungkyunkwan University, ${ }^{4}$ Department of \\ Pathology, Samsung Medical Center, Sungkyunkwan University School of Medicine, ${ }^{5}$ Center for Clinical Epidemiology, Samsung Medical \\ Center, Sungkyunkwan University, Seoul, Korea, and ${ }^{6}$ Department of Epidemiology and Medicine and Welch Center for Prevention, \\ Epidemiology and Clinical Research, Johns Hopkins Medical Institutions, Baltimore, MD, USA
}

See editorial on page 279.

Background/Aims: Recent advances in understanding the genetics of pancreatic ductal adenocarcinoma (PDAC) have led to the potential for a personalized approach. Several studies have described the feasibility of generating genetic profiles of PDAC with next-generation sequencing (NGS) of samples obtained through endoscopic ultrasound-guided tissue acquisition (EUS-TA). The aim of this study was to find the best EUS-TA approach for successful NGS of PDAC. Methods: We attempted to perform NGS with tissues from 190 patients with histologically proven PDAC by endoscopic ultrasound-guided fine-needle aspiration and endoscopic ultrasound-guided fine-needle biopsy at Samsung Medical Center between November 2011 and February 2015. The medical records of these patients were retrospectively reviewed for parameters including tumor factors (size, location, and T stage), EUS-TA factors (needle gauge [G], needle type, and number of needle passes) and histologic factors (cellularity and blood contamination). The sample used for NGS was part of the EUS-TA specimen that underwent cytological and histological analysis. Results: NGS could be successfully performed in 109 patients (57.4\%). In the univariate analysis, a large needle $G(p=0.003)$ and tumor located in the body/tail $(p=0.005)$ were associated with successful NGS. The multivariate logistic regression analysis revealed that the needle $\mathrm{G}$ was an independent factor of successful NGS (odds ratio, 2.19; 95\% confidence interval, 1.08 to 4.47 ; $p=0.031$ ).
Conclusions: The needle $\mathrm{G}$ is an independent factor associated with successful NGS. This finding may suggest that the quantity of cells obtained from EUS-TA specimens is important for successful NGS. (Gut Liver 2020;14:387394)

Key Words: Next-generation sequencing; Endoscopic ultrasound-guided tissue acquisition; Endoscopic ultrasound guided fine-needle aspiration; Endoscopic ultrasound guided fine-needle biopsy; Pancreatic ductal adenocarcinoma

\section{INTRODUCTION}

Pancreatic ductal adenocarcinoma (PDAC) is a highly lethal disease with an overall survival of only 7.0\% and ranks as the fifth highest cancer-related cause of death in the world. ${ }^{1}$ Surgical resection remains the only potentially curative therapy for patients with PDAC. However, up to 85\% patients are diagnosed at unresectable stage and need chemotherapy. ${ }^{2-4}$ Recently, several regimens showed better survival than conventional gemcitabine-based chemotherapy, but the response rate of most effective chemotherapy in PDAC is less than $50 \% .^{5-9}$ If we could select patient who would not response to a specific chemotherapy, we might choose a different regimen or offer an investigational agent according to prediction models. For this purpose, genomics and sequencing technology could be helpful to select patients who could be beneficial from a specific chemotherapy.

With development and improvement of human genomics and sequencing technology, personalized medicine of cancer

Correspondence to: Kwang Hyuck Lee

Department of Medicine, Samsung Medical Center, Sungkyunkwan University School of Medicine, 81 Irwon-ro, Gangnam-gu, Seoul 06351, Korea

Tel: +82-2-3410-3409, Fax: +82-2-3410-6983, E-mail: lkhyuck@gmail.com

Received on January 8, 2019. Revised on May 11, 2019. Accepted on May 12, 2019. Published online October 8, 2019.

pISSN 1976-2283 eISSN 2005-1212 https://doi.org/10.5009/gnl19011

Jae Keun Park and Ji Hyeon Lee contributed equally to this work as first authors.

(a) This is an Open Access article distributed under the terms of the Creative Commons Attribution Non-Commercial License (http://creativecommons.org/licenses/by-nc/4.0) which permits unrestricted non-commercial use, distribution, and reproduction in any medium, provided the original work is properly cited. 
has become a reality. ${ }^{10}$ Especially, next-generation sequencing (NGS) has been widely implemented for gene sequencing; there is great potential for NGS application in disease diagnosis, management and prognosis assessment. ${ }^{11-13}$ NGS has been successfully employed to identify novel mutations in a variety of cancers including bladder cancer, renal cell carcinoma, small cell lung cancer, prostate cancer, acute myelogenous leukemia and chronic lymphocytic leukemia. ${ }^{10}$ Recently, also in PDAC, several studies revealed mutational landscape of PDAC using NGS. However, most of studies used surgical specimens for sequence analysis. ${ }^{14-18}$ The advent of targetable therapies in PDAC has lagged behind other cancers. The reason is the lack of adequate tissue specimens for detailed sequence analysis in patients with unresectable PDAC. For patients with inoperable PDAC, a fineneedle aspirate of PDAC obtained by endoscopic ultrasoundguided fine-needle aspiration (EUS-FNA) is a useful in making a tissue diagnoses and has the potential to provide cancer DNA for sequencing analysis. ${ }^{19-21}$ Several studies have described the feasibility of NGS using endoscopic ultrasound-guided tissue acquisition (EUS-TA) specimens obtained by EUS-FNA and endoscopic ultrasound-guided fine-needle biopsy (EUS-FNB). ${ }^{22-26}$ However, the yields of NGS using EUS-TA specimen and factors of EUS-TA for successful NGS in PDAC have not been evaluated so far.

The aims of this study were to determine the yield of NGS using remaining fresh frozen EUS-TA specimens obtained from patients with PDAC and to evaluate the factors of EUS-TA for successful NGS in PDAC. This work could lead to increase in success rate of NGS and facilitate the development of personalized medicine in PDAC.

\section{MATERIALS AND METHODS}

\section{Patients}

A total of 190 patients' samples were obtained between November 2011 and February 2015 at Samsung Medical Center with informed consent from patients (Fig. 1). All of these 190 samples with pathologically confirmed PDAC using EUS-ENA and EUS-FNB were retrospectively enrolled. We performed NGS using the customized cancer panel (CancerSCAN $\left.{ }^{\mathrm{TM}}\right)^{27}$ of 190 samples from these patients to investigate genomic profile to predict the chemotherapy response in unresectable PDAC.

We investigated tumor characteristics such as location, size and stage, EUS-TA techniques such as needle size, type and number of pass, and specimen quality including cellularity and blood contamination in addition to patient's age, sex and blood test to find the best way to get samples for successful NGS. We defined cellularity and blood contamination as follows; cellularity was graded into four levels: scanty, no cellular smear; low, $<2$ clusters of malignant cells with a minimum of 10 cells; moderate, about 2 to 4 clusters of malignant cells with a minimum of 10 cells each; high, $>4$ clusters of malignant cells with a min- imum of 10 cells each. Blood contamination was also graded into four levels: none, absent blood cells; low, a few blood cells without affecting cytopathology diagnosis; moderate, partially obscured by blood cells but possible cytopathology diagnosis; high, obscured by blood cells leading to inadequate interpretation. This study was approved by the Institutional Review Board of Samsung Medical Center (IRB number: 2015-07-143).

\section{EUS-TA (EUS-FNA/EUS-FNB) procedure}

Informed consent for purpose and complication of EUS-TA was obtained from all patients prior to procedure. All EUS-TA procedures were conducted under conscious sedation by two certified endosonographers (J.K.L. and K.H.L.). A GF-UE160-AL linear EUS apparatus (Olympus, Tokyo, Japan) equipped with an Aloka ProSound SSD 5000 processor (Wallingford, CT, USA) was used in all cases. EchoTip ${ }^{\circledR}$ Ultra Endoscopic Ultrasound Needle or EndoTip ProCore ${ }^{\circledR}$ HD Ultrasound Biopsy Needle (19, 22 or 25 gauges [G]; Wilson-Cook Inc., Winston-Salem, NC, USA) were used, allowing several passes under direct endosonographic visualization to obtain sufficient cellular aspirates for cytologic analysis. The choice of needle diameter was based on the circumstances at the time. A transgastric approach was performed on lesions in the body or tail of the pancreas, and a transduodenal approach was used for lesions in the head or uncinate process. For tissue retrieval, the stylet was introduced into the needle or the needle was flushed with a 5 to $10 \mathrm{~mL}$ air-filled syringe. The extruded material was placed onto glass slides for primary gross inspection. EUS-TA specimen was placed into

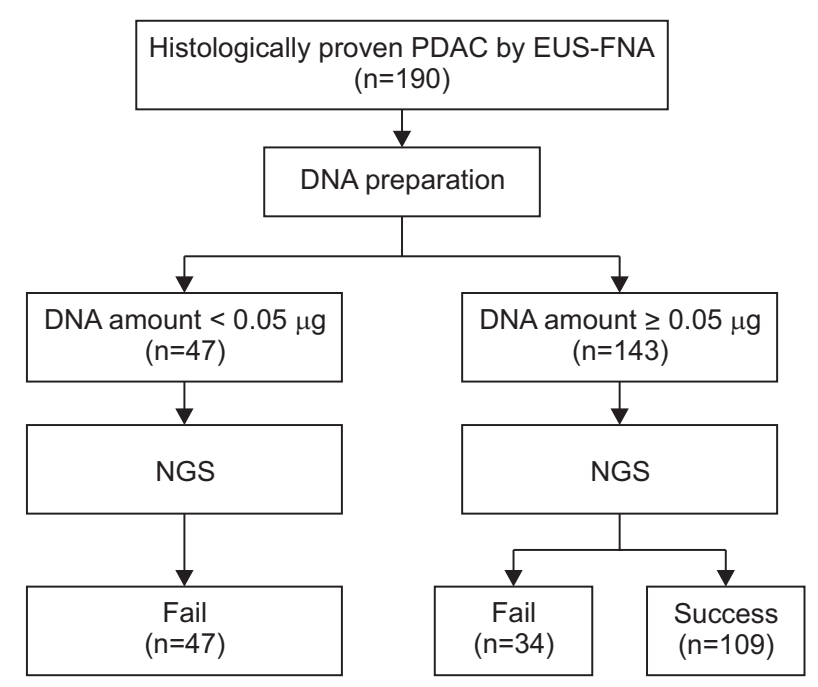

Fig. 1. A total of 190 patients were pathologically confirmed to have PDAC by EUS-TA. Among these patients, 143 patients with DNA that passed quality control testing (minimum $0.05 \mu \mathrm{g}$ ) were analyzed for their genomic profiles by targeted NGS using the CancerSCAN ${ }^{\mathrm{TM}}$ panel.

PDAC, pancreatic ductal adenocarcinoma; EUS-TA, endoscopic ultrasound-guided tissue acquisition; NGS, next-generation sequencing; EUS-FNA, endoscopic ultrasound-guided fine-needle aspiration. 
$10 \%$ formalin solution for histological analysis and/or smeared on the slide and fixed with 95\% ethanol for cytological analysis. Because an immediate on-site cytological analysis was not available, preparative procedures were performed by the examiner. After a part of EUS-TA specimen was used for cytological and histological analysis, the examiners put the remaining tissue into a tube and stored it at $-80^{\circ} \mathrm{C}$ refrigerator.

\section{Genomic extraction and DNA sequencing}

Genomic DNA from a fresh frozen sample was extracted using a QIAamp DNA mini kit (Qiagen Inc, Valencia, CA, USA). Tumor purity under 30\% was less reliable for DNA extraction and was not included in our study. Genomic DNA quality and quantity were determined using a Nanodrop 8000 UV-Vis spectrometer (Thermo Scientific Inc., Wilmington, DE, USA), Qubit 2.0 Fluorometer (Life Technologies Inc., Grand Island, NY, USA) and 2200 TapeStation Instrument (Agilent Technologies, Santa
Clara, CA, USA). After quantification of DNA amount, only samples with DNA amount of more than or equal to $0.05 \mu \mathrm{g}$ were used for sequencing. For the NGS, HiSeq 2500 sequencing platform (Illumina, San Diego, CA, USA) designed to enrich the exons of 83 genes, covering $366.2 \mathrm{~kb}$ of the human genome was used. All of these 190 samples were profiled on CancerSCAN ${ }^{\mathrm{TM}}$ version 1, which targeted 83 genes. ABL1, AKT1, AKT2, AKT3, ALK, APC, ARID1A, ARID1B, ARID2, ATM, ATRX, AURKA, AURKB, BCL2, BRAF, BRCA1, BRCA2, CDH1, CDK4, CKD6, CDKN2A, CSF1R, CTNNB1, DDR2, EGFR, EPHB4, ERBB2, ERBB3, ERBB4, EWSR1, EZH2, FBXW7, FGFR1, FGFR2, FGFR3, FLT3, GNA11, GNAQ, GNAS, HNF1A, HRAS, IDH1, IDH2, IGF1R, ITK, JAK1, JAK2, JAK3, KDR, KIT, KRAS, MDM2, MET, MLH1, MPL, MTOR, FN1, NOTCH1, NPM1, NRAS, NTRK1, PDGFRA, PDGFRB, PIK3CA, PIK3R1, PTCH1, PTCH2, PTEN, PTPN11, RB1, PET, ROS1, SMAD4, SMARCB1, SMO, SRC, STK11, SYK, TERT, TOP1, TP53, TMPRSS2, and VHL were included (Fig. 2). Suc-

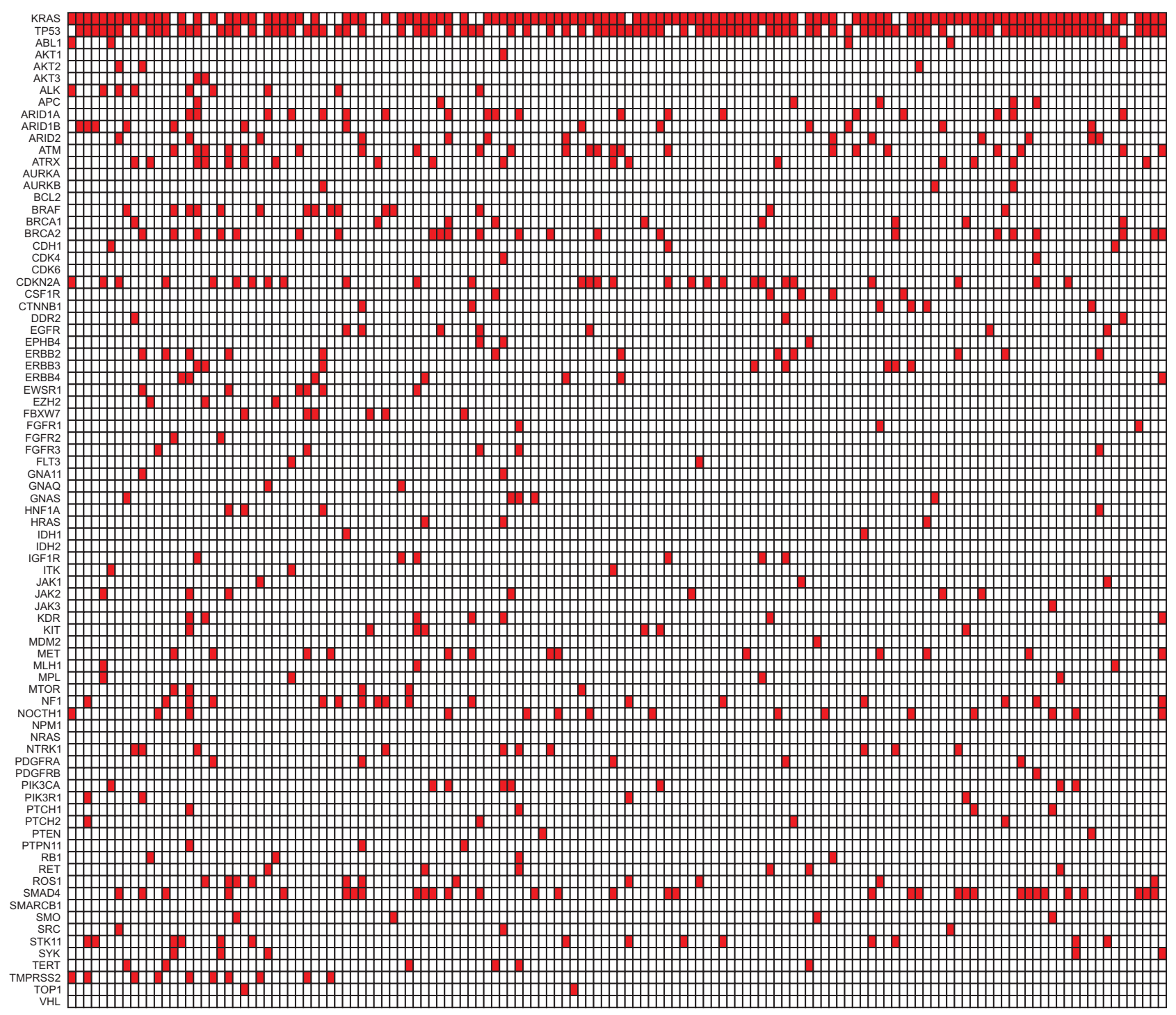

Fig. 2. Next-generation sequencing (NGS) with 83 key mutation genes for the 109 NGS-detected patients. 
cessful NGS was defined as successful gene sequencing of patients' samples using 83 genes (CancerSCAN V1, 83-gene panel at $\sim 900 x$ ).

\section{Statistical analysis}

Patient's baseline characteristics were summarized using descriptive statistics. The data were presented as the mean and standard deviation or percentages. The Student t-test and MannWhitney test were used to compare continuous variables, and the chi-square test or Fisher exact test were used to compare categorical variables. Logistic regression analysis was used to identify factors of EUS-TA for successful NGS. p-values $<0.05$ were considered to indicate statistical significance. Statistical analysis was executed using SAS version 9.4 (SAS Institute, Cary, NC, USA).

\section{RESULTS}

\section{Patient demographics and characteristics}

The demographic and baseline characteristics of patients are presented in Table 1. One-hundred twelve patients (59.0\%) were male; the mean age of the patients was 62.1 years. Average tumor size was $3.9 \mathrm{~cm}$ and $43.7 \%$ were located in the head or uncinate process and 56.3\% in body and tail. The patients with metastasis were 106 (55.8\%); the most common metastatic site was the liver.

\section{Characteristics of EUS-TA (EUS-FNA/EUS-FNB) proce- dure and histology}

Sampling methods of EUS-TA procedure are presented in Table 2. One-hundred thirty-three patients (70\%) underwent EUS-guided sampling with 19-G needle or 22-G needle and 49 patients (25.8\%) with 25-G needle, respectively. We obtained $30 \%$ of samples with EchoTip ${ }^{\circledR}$ Ultra Endoscopic Ultrasound Needle (19, 22 or 25 G; Wilson-Cook Inc) and 70\% with EndoTip ProCore ${ }^{\circledR}$ HD Ultrasound Biopsy Needle (19, 22 or 25 G; Wilson-Cook Inc). Among the 83 patients with PDAC in the head or uncinate process, 36 patients (43.4\%) underwent EUSguided sampling with $25-G$ needle. The mean number of needle passes were 3.21 times. The $73.0 \%$ of obtained EUS-TA samples showed high cellularity. Absent or low blood contamination was observed in $92.1 \%$.

\section{DNA amounts}

NGS was successfully performed in 109 of the 190 patients (57.4\%) (Fig. 1). DNA amount according to the EUS-TA needle type was not significantly different between EUS-FNA and EUSFNB ( $p=0.189$ ) (Fig. 3). On the other hand, DNA amount of 25-G EUS-TA needle was significantly smaller than that of 19- or $22-G$ needle $(p<0.001)$ (Fig. 3). DNA amount was significantly higher in NGS success group than NGS fail group $(1.42 \pm 1.57$ $\mu \mathrm{g}$ vs $0.54 \pm 1.70 \mu \mathrm{g}, \mathrm{p}<0.001)$. The success rate of NGS samples with DNA amount more than $0.05 \mu \mathrm{g}$ was $76 \%(\mathrm{n}=109 / 143)$

Table 1. Baseline Characteristics of Patients and Tumor Characteristics

\begin{tabular}{|c|c|c|c|c|}
\hline Characteristics & Total $(n=190)$ & NGS not detected $(n=81)$ & NGS detected $(\mathrm{n}=109)$ & p-value \\
\hline Age, yr & $62.1 \pm 10.6$ & $62.1 \pm 10.6$ & $62.1 \pm 10.7$ & 0.889 \\
\hline Male sex & $112(59.0)$ & 49 (43.8) & $63(56.3)$ & 0.709 \\
\hline T stage & & & & 0.473 \\
\hline $\mathrm{T} 1$ & $1(0.5)$ & $1(100)$ & 0 & \\
\hline $\mathrm{T} 2$ & $7(3.7)$ & $2(28.6)$ & $5(71.4)$ & \\
\hline T3 & $67(35.3)$ & $26(38.8)$ & $41(61.2)$ & \\
\hline $\mathrm{T} 4$ & $115(60.5)$ & $52(45.2)$ & $63(54.8)$ & \\
\hline Tumor size, $\mathrm{cm}$ & $3.9 \pm 1.7$ & $3.7 \pm 1.5$ & $4.1 \pm 1.9$ & 0.368 \\
\hline Tumor location & & & & 0.005 \\
\hline Head/uncinate process & $83(43.7)$ & $45(54.2)$ & $38(45.8)$ & \\
\hline Body/tail & $107(56.3)$ & $36(33.6)$ & $71(66.4)$ & \\
\hline Metastasis (yes) & $106(55.8)$ & 40 (37.7) & $66(62.3)$ & 0.125 \\
\hline \multicolumn{5}{|l|}{ Metastatic site } \\
\hline Liver & $67(35.3)$ & $24(35.8)$ & $43(64.2)$ & 0.161 \\
\hline Peritoneum & $35(18.4)$ & $11(31.4)$ & $24(68.6)$ & 0.138 \\
\hline Lung & $21(11.1)$ & $10(47.6)$ & $11(52.4)$ & 0.624 \\
\hline Bone & $7(3.7)$ & $4(57.1)$ & $3(42.9)$ & 0.462 \\
\hline Operation (yes) & $33(17.4)$ & $18(54.5)$ & $15(45.5)$ & 0.128 \\
\hline
\end{tabular}

Data are presented as mean \pm SD or number $(\%)$. NGS, next-generation sequencing. 
Table 2. Characteristics of the EUS-TA Procedure and Pathology Results

\begin{tabular}{|c|c|c|c|c|}
\hline Variable & Total $(\mathrm{n}=190)$ & NGS not detected $(n=81)$ & NGS detected $(n=109)$ & p-value \\
\hline Needle gauge of $\mathrm{TA}^{*}$ & & & & 0.003 \\
\hline $19 \mathrm{G}+22 \mathrm{G}^{\dagger}$ & 133 & 49 (36.8) & $84(63.2)$ & \\
\hline $25 \mathrm{G}$ & 49 & $30(61.2)$ & $19(38.8)$ & \\
\hline Needle type & & & & 0.089 \\
\hline FNA $\left(19 \mathrm{G}+22 \mathrm{G}^{\dagger} / 25 \mathrm{G}\right)^{\ddagger}$ & $57(42 / 11)$ & $19(12 / 6)$ & $38(30 / 5)$ & \\
\hline Procore $\left(19 \mathrm{G}+22 \mathrm{G}^{\dagger} / 25 \mathrm{G}\right)^{\S}$ & $133(91 / 38)$ & $62(37 / 24)$ & $71(54 / 14)$ & \\
\hline No. of needle passes & $3.21 \pm 1.20$ & $3.14 \pm 0.77$ & $3.26 \pm 1.44$ & 0.502 \\
\hline Cellularity" & & & & 0.818 \\
\hline Scanty & 2 & 0 & $2(100)$ & \\
\hline Low & 6 & $3(50)$ & $3(50)$ & \\
\hline Moderate & 43 & $18(41.9)$ & $25(58.1)$ & \\
\hline High & 138 & $59(42.8)$ & $79(57.2)$ & \\
\hline Blood contamination" & & & & 0.753 \\
\hline None & 65 & $30(46.2)$ & $35(53.8)$ & \\
\hline Low & 110 & 45 (40.9) & $65(59.1)$ & \\
\hline Moderate & 12 & $4(33.3)$ & $8(66.7)$ & \\
\hline High & 1 & 0 & $1(100)$ & \\
\hline
\end{tabular}

Data are presented as number (\%) or mean \pm SD.

EUS-TA, endoscopic ultrasound-guided tissue acquisition; NGS, next-generation sequencing; G, gauge; FNA, fine-needle aspiration.

${ }^{*}$ Eight were missing information regarding the needle gauge of EUS-TA; ${ }^{\dagger}$ Three samples obtained with a $19-\mathrm{G}$ needle were detected in NGS; ${ }^{\ddagger}$ Four were missing information regarding the needle type of EUS-TA. One was not detected in NGS, and 3 were detected in NGS; ${ }^{\S}$ Four were missing information regarding the needle type of EUS-TA, 1 was not detected in NGS, and 3 were detected in NGS; "One was missing information regarding the cellularity of EUS-TA; "Two were missing information regarding the blood contamination of EUS-TA.
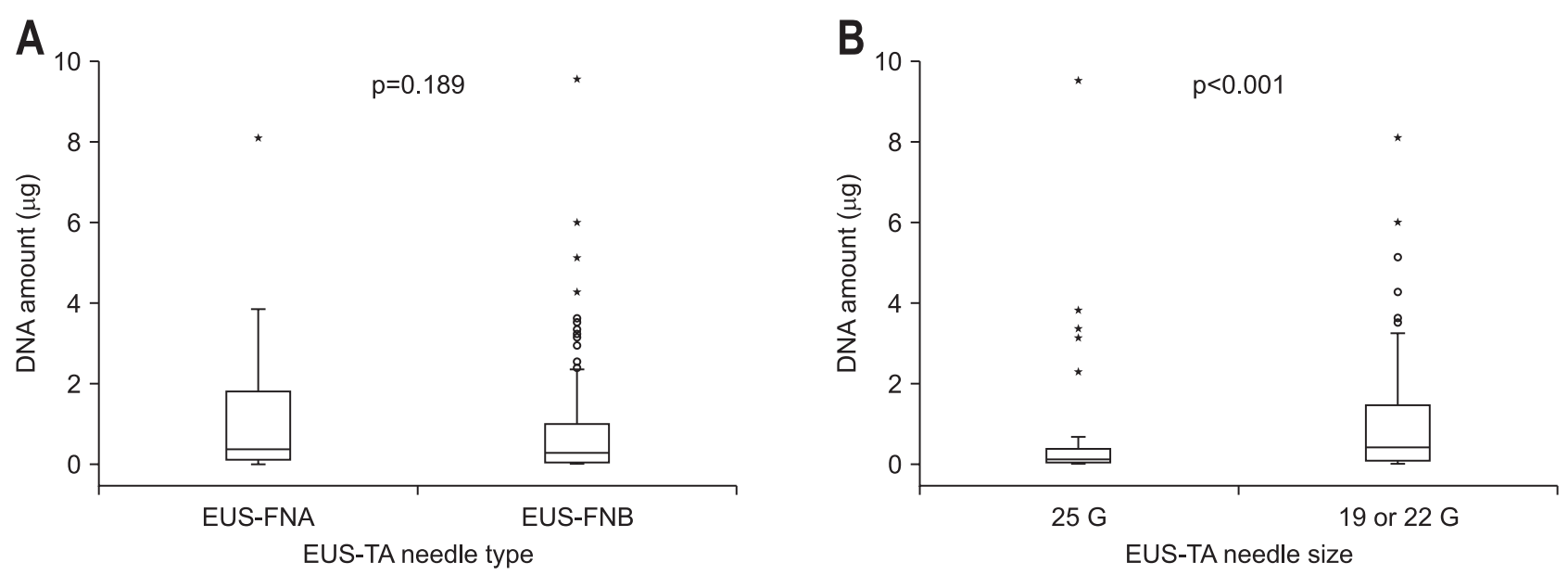

Fig. 3. The amount of DNA acquired according to the needle type and size.

EUS-TA, endoscopic ultrasound-guided tissue acquisition; EUS-FNA, endoscopic ultrasound-guided fine-needle aspiration; EUS-FNB, endoscopic ultrasound-guided fine-needle biopsy; G, gauge.

and the DNA amount of successful NGS was different statistically to those of failed NGS samples with DNA amount more than $0.05 \mu \mathrm{g}$ ( 35 vs 109: $0.17 \pm 0.41$ vs $1.42 \pm 1.57, \mathrm{p}<0.001$ ).

\section{Factors for successful NGS}

In the univariate analysis, location of tumor and size of needle were related to higher success rate of NGS analysis. NGS success rate of samples from the body/tail cancers was higher 
Table 3. Multivariate Logistic Regression Analysis

\begin{tabular}{ccc}
\hline & OR $(95 \% \mathrm{CI})$ & p-value \\
\hline Needle gauge & Reference & \\
$25 \mathrm{G}$ & $2.19(1.08-4.47)$ & 0.031 \\
$19 \mathrm{G}$ or $22 \mathrm{G}$ &
\end{tabular}

OR, odds ratio; $\mathrm{CI}$, confidence interval; $\mathrm{G}$, gauge.

than that from the head/uncinate process (66.4\% vs $45.8 \%$, $\mathrm{p}=0.005$ ). Success rate of larger $\mathrm{G}$ needle also higher than that of smaller G needle (38.8\% vs 60.9\%, p=0.003). In the chisquare analysis, there is significant relationship between the location of tumor and size of needle $(\mathrm{p}<0.005)$.

In this analysis, there were no statistically significant differences in patient demographics between NGS success group and NGS fail group. Tumor size was not significantly different between NGS success group and NGS fail group. There were no statistically significant differences in needle type and number of needle passes. There were no statistically significant differences in pancreatic cancer T staging between two groups. In multivariate logistic regression analysis (Table 3) the G of needle remained only significant factor for successful NGS (19- or 22-G needle vs 25-G needle: odds ratio, 2.19; 95\% confidence interval, 1.08 to $4.47 ; \mathrm{p}=0.031$ ).

\section{DISCUSSION}

We investigated the yield of targeted deep sequencing NGS using remaining fresh frozen specimens after EUS-TA of PDAC and evaluated the factors for successful NGS. There were several previous studies about feasibility of NGS using TA specimens obtained by EUS-FNA and EUS-FNB. However, in these studies most of them analyzed mixed specimen of various tissue. Plus, they did not mention the factors for successful NGS. To the best of the author's knowledge, this is the largest study of targeted NGS using EUS-TA specimen in PDAC and a first study reporting about the factors of EUS-TA for successful NGS.

In our study, NGS was successfully performed in 109 of the 190 patients (57.4\%) and the G of needle was significant factor for successful NGS. Previous few studies reported the yield of NGS using FNA specimens obtained from patients with PDAC. Young et al. ${ }^{25}$ reported that genomic profiles were successfully obtained from 23 of 23 (100\%) pancreatic FNA using NGS. They used formalin-fixed, paraffin-embedded FNA specimen which contained a minimum of 15,000 total benign and malignant cells and yielded the minimum $50 \mu \mathrm{g}$ of DNA. In this study, we used remaining fresh frozen samples which were collected without intention for NGS analysis after clinical diagnostic test. So, some of the patients were too small amount for NGS. The yield of NGS can improve by applying the result of this study.

In univariate analysis tumors in body/tail showed significant higher NGS success rate than those in head/uncinate process
( $\mathrm{p}=0.005)$. A transgastric approach could be performed on lesions in the body or tail of the pancreas, otherwise, a transduodenal approach must be used for lesions in the head or uncinate process. This result could be explained by difference of accessibility, because tumors in head or uncinate process are difficult to approach. We also found that 19- or 22-G needle showed higher NGS success rate compared with $25-G$ needle $(p=0.003)$.

In multivariate analysis, the $G$ of needle remained only significant factor for successful NGS. This finding suggests that the quantity of cells from EUS-TA specimens is important for successful NGS. In our study, there were 34 patients who had DNA amounts more than $0.05 \mu \mathrm{g}$ but failed NGS. When we reviewed these patients' samples, 32 samples had DNA amounts less than $0.15 \mu \mathrm{g}$. Only two samples among 34 samples had DNA amount more than $0.15 \mu \mathrm{g}$. These two samples had the problem of DNA quality. To put it another way, there was no patient who had DNA amounts less than $0.15 \mu \mathrm{g}$ in NGS success group. Success or failure of NGS could be explained based on DNA amounts. Furthermore, DNA amount according to the EUS-TA needle size was significantly different $(\mathrm{p}<0.001)$ (Fig. 3). The smaller the needle size, the smaller the amount of DNA obtained. However, the EUS-TA needle type was not related to the amount of DNA $(p=0.189)$ (Fig. 3). Also there were no statistically significant differences in needle type $(\mathrm{p}=0.089)$. Our results were consistent with recent meta-analysis. ${ }^{28}$ Wang et al. showed no significant difference between EUS-FNA and EUS-FNB with diagnostic accuracy, sample adequacy, and in the rate of histologic core tissue procurement.

We found no difference of NGS success rate according to degree of cellularity. Several previous studies reported that high cellularity provides an excellent chance of successful sequencing. Gleeson et al. ${ }^{29}$ used adrenal metastasis cytology slides for NGS and reported that slides with low, moderate and high cellularity underwent successful NGS 22\%, 25\% and 91\% of the time. Valero et $a l .^{20}$ showed in vitro study which simulated cellularity using mixture of human PDAC cell line Panc1 and the cancer-associated fibroblast cell line CAF35. In this study, the progressive decrease in Panc1 cellularity corresponded to a stepwise reduction in mutant allele frequency. In the first study, they used adrenal metastasis cytology slides. In the second study, the study was not in vivo study but in in vitro study. So, it is difficult to compare their results to our study. However, we consider PDAC is relatively hypocellular, so the quantity of cells may be more important factor for successful NGS than the cellularity of tumor. For this, future prospective in vivo study will be needed to reveal the relation between degree of cellularity and success rate of NGS.

Our study has several limitations. First, because the sample for NGS after a part of EUS-TA specimen was used for cytological and histological analysis, our study used the sample with the potential for selection bias. Despite the anticipated selection bias, this is an article showing the importance of the quantity 
of cells from EUS-TA specimens for successful NGS. This may be the basis of using a larger $\mathrm{G}$ of a needle when acquiring a specimen for NGS. Second, because this study is retrospective study, there were some missing data collecting variables like $G$ of needle, needle type and histology. Third, two different endosonographers were involved in performing the EUS-FNA and EUS-FNB procedure, so all conditions were not identical.

In summary, in this first study about the factors of EUS-TA for NGS, we found that the yield of NGS using EUS-TA specimen in PDAC was 57.4\% and the G of needle was important factor for successful NGS. Understanding the factors for adequate NGS analysis of EUS-TA may help further studies about precision medicine in pancreatic cancer. This enables us to discover the predictive marker and invent the new effective target therapy for pancreatic cancer in near future. The development of personalized medicine will lead to improvement of treatment outcome and prognosis in highly lethal pancreatic cancer.

\section{CONFLICTS OF INTEREST}

No potential conflict of interest relevant to this article was reported.

\section{AUTHOR CONTRIBUTIONS}

Conception and design, and/or acquisition of data, and/or analysis and interpretation of data: J.K.P., J.H.L., D.H.N., K.H.L., K.T.J., J.C. Drafting of the manuscript; critical revision of the manuscript for important intellectual: J.K.P., K.T.L., J.K.L., K.H.L.

\section{ORCID}

Jae Keun Park

Ji Hyeon Lee

Dong Hyo Noh

Joo Kyung Park

Kyu Taek Lee

Jong Kyun Lee

Kwang Hyuck Lee

Kee-Taek Jang

Juhee Cho https://orcid.org/0000-0002-8323-9660 https://orcid.org/0000-0001-8035-5634 https://orcid.org/0000-0001-6431-0890 https://orcid.org/0000-0002-9652-5287 https://orcid.org/0000-0003-2233-3511 https://orcid.org/0000-0002-1298-0494 https://orcid.org/0000-0002-5558-0415 https://orcid.org/0000-0001-7987-4437 https://orcid.org/0000-0001-9081-0266

\section{REFERENCES}

1. Jemal A, Siegel R, Xu J, Ward E. Cancer statistics, 2010. CA Cancer J Clin 2010;60:277-300.

2. Mayo SC, Gilson MM, Herman JM, et al. Management of patients with pancreatic adenocarcinoma: national trends in patient selection, operative management, and use of adjuvant therapy. J Am Coll Surg 2012;214:33-45.

3. Mayo SC, Austin DF, Sheppard BC, Mori M, Shipley DK, Billingsley KG. Adjuvant therapy and survival after resection of pancreatic adenocarcinoma: a population-based analysis. Cancer 2010;116:2932-2940.

4. Kim YT. Chemotherapy for pancreatic cancer. Korean J Gastroenterol 2008;51:111-118.

5. Peyvandi F, Scully M, Kremer Hovinga JA, et al. Caplacizumab for acquired thrombotic thrombocytopenic purpura. N Engl J Med 2016;374:511-522.

6. Moore MJ, Goldstein D, Hamm J, et al. Erlotinib plus gemcitabine compared with gemcitabine alone in patients with advanced pancreatic cancer: a phase III trial of the National Cancer Institute of Canada Clinical Trials Group. J Clin Oncol 2007;25:1960-1966.

7. Conroy T, Desseigne F, Ychou M, et al. FOLFIRINOX versus gemcitabine for metastatic pancreatic cancer. N Engl J Med 2011;364:1817-1825.

8. Burris HA 3rd, Moore MJ, Andersen J, et al. Improvements in survival and clinical benefit with gemcitabine as first-line therapy for patients with advanced pancreas cancer: a randomized trial. J Clin Oncol 1997;15:2403-2413.

9. Sadot E, Doussot A, O'Reilly EM, et al. FOLFIRINOX induction therapy for stage 3 pancreatic adenocarcinoma. Ann Surg Oncol 2015;22:3512-3521.

10. Guan YF, Li GR, Wang RJ, et al. Application of next-generation sequencing in clinical oncology to advance personalized treatment of cancer. Chin J Cancer 2012;31:463-470.

11. Mardis ER. Applying next-generation sequencing to pancreatic cancer treatment. Nat Rev Gastroenterol Hepatol 2012;9:477-486.

12. Crane $\mathrm{CH}$, Iacobuzio-Donahue CA. Keys to personalized care in pancreatic oncology. J Clin Oncol 2012;30:4049-4050.

13. Humphris J, Chang DK, Biankin AV. Inherited susceptibility to pancreatic cancer in the era of next-generation sequencing. Gastroenterology 2015;148:496-498.

14. Waddell N, Pajic M, Patch AM, et al. Whole genomes redefine the mutational landscape of pancreatic cancer. Nature 2015;518:495501.

15. Jones S, Zhang X, Parsons DW, et al. Core signaling pathways in human pancreatic cancers revealed by global genomic analyses. Science 2008;321:1801-1806.

16. Villarroel MC, Rajeshkumar NV, Garrido-Laguna I, et al. Personalizing cancer treatment in the age of global genomic analyses: PALB2 gene mutations and the response to DNA damaging agents in pancreatic cancer. Mol Cancer Ther 2011;10:3-8.

17. Iacobuzio-Donahue CA, Fu B, Yachida S, et al. DPC4 gene status of the primary carcinoma correlates with patterns of failure in patients with pancreatic cancer. J Clin Oncol 2009;27:1806-1813.

18. Biankin AV, Waddell N, Kassahn KS, et al. Pancreatic cancer genomes reveal aberrations in axon guidance pathway genes. Nature 2012;491:399-405.

19. Lee JK, Lee KT, Choi ER, et al. A prospective, randomized trial comparing 25-gauge and 22-gauge needles for endoscopic ultrasound-guided fine needle aspiration of pancreatic masses. Scand J Gastroenterol 2013;48:752-757.

20. Valero V 3rd, Saunders TJ, He J, et al. Reliable detection of so- 
matic mutations in fine needle aspirates of pancreatic cancer with next-generation sequencing: implications for surgical management. Ann Surg 2016;263:153-161.

21. Lee JK, Choi JH, Lee KH, et al. A prospective, comparative trial to optimize sampling techniques in EUS-guided FNA of solid pancreatic masses. Gastrointest Endosc 2013;77:745-751.

22. Gleeson FC, Kipp BR, Levy MJ, et al. Lung cancer adrenal gland metastasis: optimal fine-needle aspirate and touch preparation smear cellularity characteristics for successful theranostic nextgeneration sequencing. Cancer Cytopathol 2014;122:822-832.

23. Karnes HE, Duncavage EJ, Bernadt CT. Targeted next-generation sequencing using fine-needle aspirates from adenocarcinomas of the lung. Cancer Cytopathol 2014;122:104-113.

24. Kubota Y, Kawakami H, Natsuizaka M, et al. CTNNB1 mutational analysis of solid-pseudopapillary neoplasms of the pancreas using endoscopic ultrasound-guided fine-needle aspiration and nextgeneration deep sequencing. J Gastroenterol 2015;50:203-210.

25. Young G, Wang K, He J, et al. Clinical next-generation sequenc- ing successfully applied to fine-needle aspirations of pulmonary and pancreatic neoplasms. Cancer Cytopathol 2013;121:688-694.

26. de Biase D, Visani M, Baccarini P, et al. Next generation sequencing improves the accuracy of KRAS mutation analysis in endoscopic ultrasound fine needle aspiration pancreatic lesions. PLoS One 2014;9:e87651.

27. Lee JY, Kim SY, Park C, et al. Patient-derived cell models as preclinical tools for genome-directed targeted therapy. Oncotarget 2015;6:25619-25630

28. Wang J, Zhao S, Chen Y, Jia R, Zhang X. Endoscopic ultrasound guided fine needle aspiration versus endoscopic ultrasound guided fine needle biopsy in sampling pancreatic masses: a meta-analysis. Medicine (Baltimore) 2017;96:e7452.

29. Gleeson FC, Kipp BR, Kerr SE, et al. Characterization of endoscopic ultrasound fine-needle aspiration cytology by targeted next-generation sequencing and theranostic potential. Clin Gastroenterol Hepatol 2015;13:37-41. 